\title{
Perfil dos usuários de benzodiazepínicos que frequentam uma drogaria da região Noroeste do Rio Grande do Sul
}

\author{
Profile of benzodiazepine users attending a drugstore \\ in the Northwest region of Rio Grande do Sul
}

\author{
Layza Kowalski ${ }^{1}$ \\ Marília Salet Schneider ${ }^{2}$ \\ Izabel Almeida Alves ${ }^{3}$
}

\section{RESUMO}

Objetivo: Traçar o perfil dos usuários de benzodiazepínicos, por meio da coleta de dados em forma de entrevista guiada por questionário nos meses de agosto a outubro do ano de 2017 no município de Cerro Largo. Metodologia:Tratase de um estudo transversal, observacional, descritivo e prospectivo que envolveu usuários de benzodiazepínicos de ambos os sexos e idades. Resultados: A maioria dos entrevistados (68\%) pertencia ao sexo feminino, com idade média dos pacientes do sexo feminino foi de 59,7 \pm 17,0 anos. Já a idade média dos pacientes do sexo masculino foi de 61,7 $\pm 12,4$. 0 tempo médio de uso foi de 1 a 3 anos e o medicamento mais utilizado foi o clonazepam 2,5 mg/ml. Entre os pacientes entrevistados, 52\% receberam prescrição de um médico clínico geral. Conclusão: 0 estudo sugere a ocorrência de uso indevido desta classe de medicamentos por períodos longos. Salienta-se que a dispensação farmacêutica deve assumir papel complementar ao serviço médico para maior resolutividade do tratamento farmacológico independente da faixa etária do usuário, com orientações quanto ao uso, risco de dependência e efeitos adversos, além de auxiliar no cuidado do paciente através pela relação paciente e prescritor.

\section{PALAVRAS-CHAVE}

Benzodiazepínicos; Dispensação farmacêutica; Atenção Farmacêutica.

\footnotetext{
${ }^{1}$ Graduanda do curso de Farmácia, da Universidade Regional Integrada do Alto Uruguai e das Missões - URI.

${ }^{2}$ Farmacêutica, Pós-Graduada em Prescrição Farmacêutica e Farmácia Clínica pela Universidade Regional Integrada do Alto Uruguai e das Missões- Santo Ângelo - URI Campus.

${ }^{3}$ Farmacêutica, Doutora em Ciências Farmacêuticas pela Universidade Federal do Rio Grande do Sul, RS.
} 


\section{ABSTRACT}

Objective: To trace the profile of benzodiazepine users through the collection of data in the form of an interview questionnaire. The questionnaire was given from the months of August to October of 2017 in the municipality of Cerro Largo. Methodology: This is a cross-sectional, observational, descriptive, and prospective study which involved users of benzodiazepines of both sexes and ages. Results: Most of the interviewees were female (68\%) with a mean age of $59.7 \pm 17.0$ years. The mean age of male patients was $61.7 \pm 12.4$. The mean time of use was 1 to 3 years and the most commonly used drug was clonazepam at a concentration of $2.5 \mathrm{mg} / \mathrm{ml}$. Among patients, $52 \%$ received a prescription from a general practitioner. Conclusion: The study suggests misuse of this class of drugs for long periods of time. It should be noted that the pharmaceutical service should assume a complementary role to the medical service for a better result in pharmaceutical treatment independent of the age range of the user; through guidance on use, risk of dependence, and adverse effects; besides assisting in patient care through the patient and prescriber relationship.

\section{KEYWORDS}

Benzodiazepines; Pharmaceutical Dispensary; Pharmaceutical Attention. 


\section{INTRODUÇÃO}

Os Benzodiazepínicos (BDZs) são fármacos depressores do sistema nervoso central (SNC) com atividade ansiolítica, que começaram a ser utilizados na década de 60 (ORLANDI; NOTO, 2005). Este grupo se caracteriza pela ação no sistema de neurotransmissão do ácido gama-amino-butírico (GABA), que é o principal sistema de neurotransmissão inibitória do SNC. Os principais efeitos adversos envolvem diminuição da cognição, amnésia anterógrada, sedação, redução da coordenação e aumento do risco de acidentes (SOUZA et al., 2013)

Seus principais efeitos terapêuticos são a sedação, hipnose e relaxamento muscular. Dentre as principais aplicações clínicas encontram-se o tratamento da ansiedade generalizada, fobias, distúrbios do sono, convulsões, espasmos musculares involuntários, dependência de álcool e outras drogas de abuso (PRADO FILHO, 2011). Para o alívio da ansiedade generalizada é recomendado seu uso em curto prazo (duas a quatro semanas somente), em distúrbios de pânico resistentes ao tratamento com antidepressivos e na opção de terapia adjunta com antidepressivos, com o objetivo de evitar piora de sintomas. Ademais, pode ser utilizado para insônia apenas quando for grave, incapacitante e estiver provocando sofrimento extremo ao paciente. São indevidamente usados para sintomas relacionados ao estresse, infelicidade, depressão e psicose (BARRETO, 2014).

Embora sejam fármacos aplicados para tratamento de diversas patologias do SNC, o uso prolongado de BDZs apresenta riscos, dentre eles um dos mais importantes é a ocorrência de dependência. Períodos de 4 a 6 semanas pode levar ao desenvolvimento de tolerância, abstinência e dependência, sendo assim devem ser usados por um período curto sob supervisão médica rigorosa (CASTRO; LARANJEIRA, 2000)

A causa da dependência é explicada por fatores que incluem variáveis relacionadas ao fármaco (maiores dosagens, uso prolongado, tempo de meia vida curto, parada brusca da utilização) e relacionadas ao paciente (comorbidade entre depressão e ansiedade, neurose, antecedentes de ataques de pânico e abuso de álcool ou outras substâncias (WANDERLEY; SANTOS, 2015). A instalação da dependência pode ser evitada pelos prescritores através do uso de dosagens mínimas e por períodos de tratamento o mais curto possível e pela seleção cuidadosa do paciente, evitando aqueles com propensão à drogadição (MARQUES, 2015).

Os sinais e sintomas físicos da síndrome de abstinência incluem: ataxia, hiperatividade autonômica (p. ex: sudorese ou taquicardia acima de 100 bpm.), náuseas e vômitos, tremores grosseiros das mãos, língua e pálpebras, hipersensibilidade a luz sons e odores, cefaléia, tontura, letargia, gosto metálico. Os sintomas psíquicos incluem: alucinações visuais, ansiedade, delírios, agitação psicomotora, ataques de pânico, prejuízos da memória, dificuldade de concentração, pesadelos (TAMELINI, 2012).

Os BZDs estão entre os medicamentos mais utilizados mundialmente, havendo estimativas de que entre 1 a 3\% de toda a população já os tenha consumido regularmente por mais de um ano (BARRETO, 2014). Segundo Nordon, (2009) no Brasil, o consumo de benzodiazepínicos é de aproximadamente $4 \%$ da população, sendo a terceira classe de drogas mais prescritas no país (NORDON, 2009).

De acordo com Paprocki, o consumo crescente de benzodiazepínicos pode ser resultado de um período particularmente turbulento que caracteriza as últimas décadas da humanidade. A diminuição progressiva da resistência da humanidade para tolerar tanto estresse, a introdução profusa de novas drogas e a pressão propagandística crescente por parte da indústria farmacêutica ouainda, hábitos de prescrição inadequada por parte dos prescritores podem ter contribuído para o aumento da procura pelos benzodiazepínicos, ampliando a possibilidade de uso abusivo e as suas consequências, como as reações adversas, intoxicações e dependência (PAPROCKI, 1990).

Outros problemas de saúde estão relacionados ao uso de BZD, principalmente nos idosos, como o aumento das taxas de acidentes, quedas e fraturas, e há evidências de que doses terapêuticas podem prejudicar as funções cognitivas em idosos, mesmo após a interrupção do medicamento (RUFF, 2000).

A atenção farmacêutica é importante para os usuários de benzodiazepínicos ao orientar e colocar o paciente a par de todos os possíveis riscos diante da utilização desses medicamentos. Assim, o farmacêutico contribui para o uso racional dos medicamentos, 
trabalha em prol da promoção da saúde e da vida e o paciente terá consciência dos riscos e benefícios da terapêutica que ele fará uso (MAGALHÃES, 2016).

0 presente trabalho teve como objetivo avaliar o perfil de um grupo de usuários de benzodiazepínicos em uma drogaria do município de Cerro Largo, bem como identificar o possível uso abusivo e discutir a utilização e a promoção do uso racional dos medicamentos prescritos, evidenciando a contribuição do farmacêutico como integrante da equipe multiprofissional de saúde.

\section{MATERIAIS E MÉTODOS}

\section{Delineamento do Estudo}

Trata-se de um estudo transversal, observacional, descritivo e prospectivo com usuários de benzodiazepínicos durante os meses de agosto a outubro do ano de 2017.

\section{Metodologia}

0 estudo foi realizado em uma drogaria privada na cidade de Cerro Largo, localizado na região Noroeste do Rio Grande do Sul, com uma população estimada de 14.069 habitantes, conforme dados do IBGE 2017.

A amostra, foi obtida por conveniência e os pacientes foram convidados a participar da pesquisa na ocasião que procuraram o estabelecimento para adquirir pelo menos um medicamento benzodiazepínico. Os dados foram coletados por meio de um questionário com perguntas abertas e fechadas, realizadas pelo farmacêutico local. Aplicou-se o questionário pela técnica de entrevista, em que se verificou o gênero, idade, escolaridade, patologias relatadas pelo paciente, uso de outros medicamentos, informações que possuem sobreo medicamento, tempo de uso dobenzodiazepínico, especialidademédicaqueoprescreveu,sejásuspenderam o uso ou reduziram a dose e quais os sintomas.

As informações obtidas foram demonstradas através da análise descritiva. Os dados foram organizados em um banco de dados no Excel@ 2011. A análise descritiva foi realizada pela apresentação dos resultados em frequências (variáveis qualitativas), média e desvio padrão (variáveis quantitativas).

\section{Aspectos Éticos}

A pesquisa foi aprovada pelo Comitê de Ética em Pesquisa da Universidade Regional Integrada do Alto Uruguai e das Missões (URI - Campus Santo Ângelo) sob o no 038867/2017, e aos indivíduos que aceitaram participar da pesquisa foi apresentado o Termo de Consentimento Livre e Esclarecido, o qual foi assinado previamente à coleta das informações.

\section{RESULTADOS E DISCUSSÃO}

Foram entrevistados 25 pacientes, entre eles, 17 do sexo feminino (68\%) e 8 do sexo masculino (32\%). A idade média dos pacientes do sexo feminino foi de 59,7 $\pm 17,0$ anos. Já a idade média dos pacientes do sexo masculino foi de $61,7 \pm 12,4$. Sendo que a idade mínima dos usuários foi 26 anos e a máxima 90 anos.

Com o presente estudo pode-se observar que o uso de medicamentos benzodiazepínicos é maior na população feminina e aumenta com a idade (figura 1)

Figura 1. Distribuição dos usuários de benzodiazepínicos entrevistados na drogaria, conforme faixa etária.

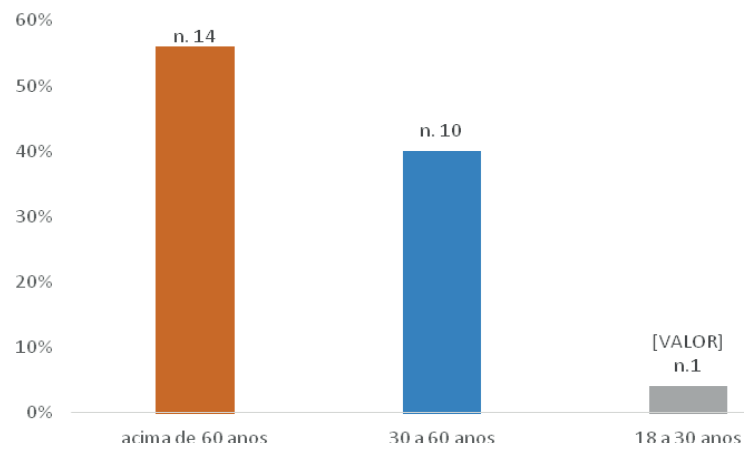

Os dados deste estudo são semelhantes a maior parte dos estudos conduzidos em outros países, em que se verifica que o consumo é mais prevalente nas mulheres e nas faixas etárias mais elevadas (CARVALHO; DIMENSTEIN, 2004). 0 aumento da idade é fator de risco estabelecido,nãoapenasparaouso debenzodiazepínicos, mastambém,paraousoprolongadodebenzodiazepínicos (HUF; LOPES; ROZENFELD, 2000).

As mulheres geralmente preocupam-se mais com a saúde, procuram com maior frequência a assistência médica, descrevem com mais facilidade os problemas físicos e psicológicos, o que aumenta a probabilidade de 
receberem e aceitarem a prescrição de psicotrópicos. Adicionalmente, o gênero feminino é mais afetado por problemas de saúde não-fatais, verificando-se maior tendência de procura por atendimento médico e de prescrição de psicotrópicos (NOIA et al., 2012).

0 tempo de uso de benzodiazepínicos variou de 3 meses a 25 anos (figura 2). 0 tempo médio de uso foi de 1 a 3 anos, mostrando que há usos crônicos com risco de instalação de dependência e casos recentes de uso.

Embora as recomendações para o uso de benzodiazepínicos com prescrição sugerem que a duração se limite a algumas semanas, é conhecido o uso desses medicamentos por meses, anos, ou até décadas, mesmo que as evidências demonstrem que seus benefícios podem diminuir com o tempo, enquanto o potencial para efeitos adversos permanece (AUTHIER et al., 2009).

Figura 2. Tempo de uso dos medicamentos benzodiazepínicos pelos pacientes atendidos na drogaria do estudo.

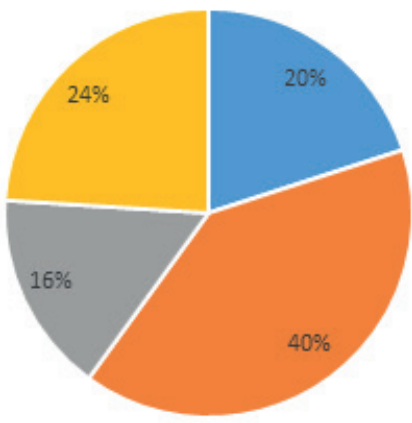

- menos de 1 ano = 1 a 3 anos = 6 a 10 anos = maisde 10 anos

A Associação Brasileira de Psiquiatria e Neurologia recomenda que se deve evitar seu uso por mais de três meses, tempo considerado crônico, pois isto aumenta a possibilidade do desenvolvimento de tolerância e dependência. 0 tempo de uso irá depender dos sintomas a serem tratados: para casos de insônia, o uso deve ser restrito há alguns dias, alternados ou não, e não deve ultrapassar duas semanas. Para o tratamento de ansiedade idealé o uso concomitante de antidepressivos e de tratamento psicoterápico (BRAGA et al., 2011). A tolerância (dose inicial não produz o mesmo efeito ou é necessário aumentar as doses para atingir o efeito inicial) se desenvolve especialmente para os efeitos sedativos, permanecendo algum efeito ansiolítico e efeitos anestésicos. 0 paciente pode não experimentar síndrome de abstinência, já que continuará o uso de modo indefinido, a não ser que fique sem receita por algum incidente, onde poderá enfrentar os sinais e sintomas da síndrome de abstinência, mas logo poderá considerar que são sinais de ansiedade subjacente e que, não deveria parar a medicação (ASSOCIAÇÃO BRASILEIRA DE PSIQUIATRIA E NEUROLOGIA, 2013).

Orlandi e Noto (2005), partindo da perspectiva dos profissionais de saúde sobre a prescrição, dispensação e o uso de BZD, encontraram um período de uso geralmente superior a dois 2 anos (ORLANDI; NOTO, 2005).

$\mathrm{Na}$ pesquisa de Silva e Oliveira grande parte dos usuários (8 - 44,4\%) utiliza a medicação entre 1 e 3 anos e de forma contínua, confirmando o resultado desta pesquisa, sendo que, para Auchewski e colaboradores (2004) o uso de BDZ por mais de 12 meses representa riscos de $25 \%$ a $40 \%$ para o usuário, sendo assim, os participantes deste estudo podem fomentar esta estatística (SILVA, 2012).

Os BZDs têm potencial de indução de dependência, $50 \%$ dos pacientes que usam BZDs por mais de 12 meses evoluem com síndrome de abstinência. Os sintomas começam progressivamente dentro de 2 a 3 dias após a parada de BZDs de meia-vida curta e de 5 a 10 dias após a parada de BZDs de meia-vida longa, podendo também ocorrer após a diminuição da dose (MARQUES; TIENGO; NOGUEIRA, 2013).

A percepção da dependência foi relatada pelas usuárias como queixa do que sentem quando estão sem a medicação, ou pela antecipação da falta do medicamento, ou ainda pelo relato de não conseguir viver sem a medicação. Esses dados corroboram o estudo de Anthierens et al., que relatam que a dependência foi observada mesmo em doses terapêuticas, notando-se que o paciente carregava comprimidos consigo, e que usava uma dose superior ao habitual mediante antecipação de situações estressantes, além de relato dos pacientes que tinham "medo" que os sintomas (como insônia ou ansiedade) voltassem se eles interrompessem a medicação (SOUZA et al., 2013).

De acordo com Huf, Lopes \& Rozenfeld (2000) médicos e usuários afirmam que os BDZs são os medicamentos mais difíceis de interromper o uso, além disso, metade das pessoas que interrompe um tratamento com esses medicamentos, reinicia o uso após um ano (HUF; LOPES; ROZENFELD, 2000).

Adependênciadosbenzodiazepínicoséintensificada nos idosos. No entanto, a dependência de 
benzodiazepínicos nem sempre é enfatizada, existindo a não-notificação nos prontuários médicos desse tipo de dependência (MENDONÇA, 2005). Entre as variáveis sócio demográficas, os baixos níveis de renda e escolaridade são apontados em alguns estudos; e, entre os problemas de saúde, a presença de insônia e queixas ósteoarticulares, musculoesqueléticas egastrintestinais têm sido associadas ao uso prolongado de benzodiazepínicos (CASTRO et al., 2013).

$\mathrm{Na}$ figura 3, pode-se verificar que entre os entrevistados, $32 \%$ possuíam ensino fundamental incompleto. Estudo realizado no sul do Brasil demonstra que o consumo de psicotrópicos tem como prevalência o grau de escolaridade abaixo da quarta série
(RODRIGUES; FACCHINI; LIMA, 2006). Em outro estudo, em uma região do Rio de Janeiro, mostrou que menores níveis de renda e escolaridade, também se mantiveram associados positivamente com o uso de psicofármacos (ALMEIDA et al., 1994). No estudo de Nordon, no ano de 2010, sobre características da população usuária de benzodiazepínicos de uma Unidade de Saúde, concluiu que vários fatores geram uma situação perigosa na atenção primária: pessoas mais velhas, de menor escolaridade e, portanto, com menor informação, e provavelmente com menor renda, estão sujeitas a um uso maior de medicamentos potencialmente causadores de dependência, com efeitos colaterais orgânicos e mentais importantes (NORDON et al., 2010).

Figura 3. Nível de Escolaridade dos participantes da pesquisa.

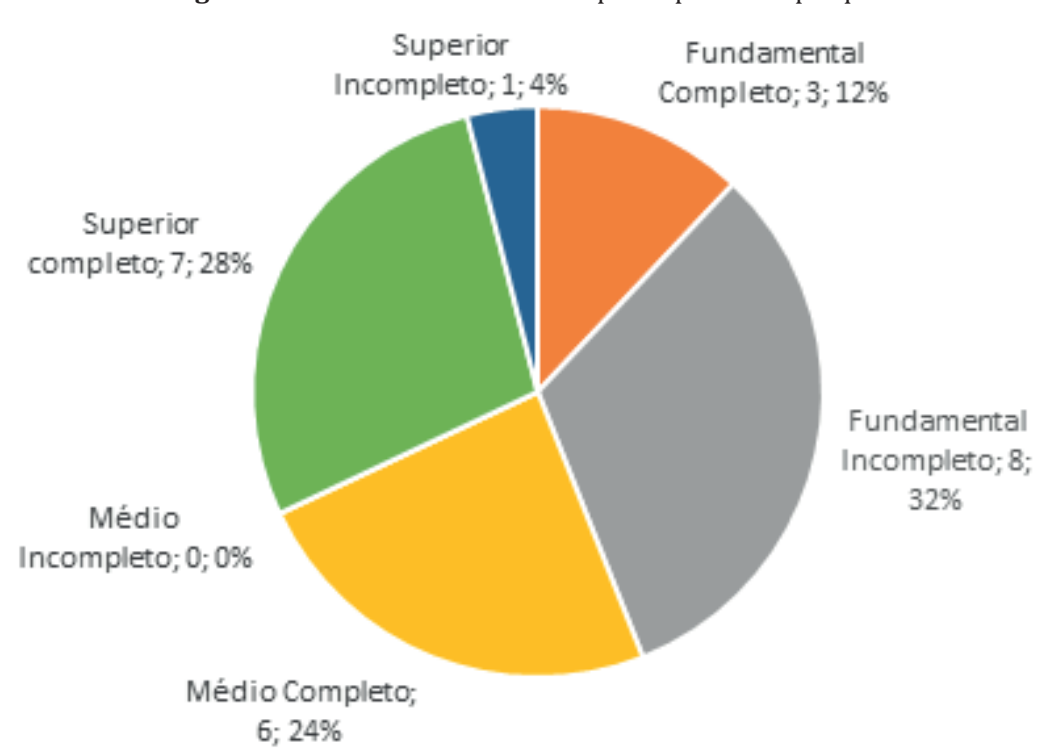

Tabela 1. Descrição dos motivos da utilização dos Benzodiazepínicos pelos pacientes entrevistados.

\begin{tabular}{|c|c|c|}
\hline Motivo & $\mathrm{n}$ & $\%$ \\
\hline Insônia & 6 & 24 \\
\hline Ansiedade, stress, cansaço e insônia & 2 & 8 \\
\hline Ansiedade & 2 & 8 \\
\hline Síndrome do pânico & 1 & 4 \\
\hline Doença & 3 & 12 \\
\hline Depressão & 3 & 12 \\
\hline Depressão e insônia & 1 & 4 \\
\hline $\begin{array}{l}\text { Depressão, Síndrome do Pânico, Transtorno de Personalidade Borderline, Stress, Ansiedade } \\
\text { e Tremores }\end{array}$ & 1 & 4 \\
\hline Nervosismo, insônia e ansiedade & 2 & 8 \\
\hline Insônia após falecimento de esposa/esposo & 2 & 8 \\
\hline Ansiedade onde aumenta a pressão arterial e palpitação & 2 & 8 \\
\hline
\end{tabular}


Foi pesquisado os motivos pelos quais as pacientes faziam uso de benzodiazepínicos (tabela 1). Três pacientes começaram seu uso após doença (uma delas após tratamento para câncer de útero, outro paciente após cirurgia de coluna sendo que não pode trabalhar, $\mathrm{e}$ um após acidente vascular cerebral). Uma das pacientes entrevistadas relatou que "precisa saber que tem o medicamento em casa ou na bolsa, sente medo e ansiedade de não ter o medicamento quando precisar".

0 medicamento mais utilizado foi o clonazepam 2,5 $\mathrm{mg} / \mathrm{ml}$ em solução oral, seguido de alprazolam 0,5 mg sob forma de comprimidos para via oral (tabela 2).

Tabela 2. Relação de medicamentos benzodiazepínicos utilizados pelos pacientes da pesquisa.

\begin{tabular}{lll}
\hline Medicamento & $\mathrm{n}$ & $\%$ \\
\hline Alprazolam 0,25 mg CP VO & 3 & 12 \\
Alprazolam 0,5 mg CP VO & 4 & 16 \\
Alprazolam 1 mg CP VO & 2 & 8 \\
Alprazolam 2mg + Clonazepam 2 mg CP VO & 1 & 4 \\
Bromazepam 6 mg CP VO & 3 & 12 \\
Clonazepam 0,25 mg CP VO & 3 & 12 \\
Clonazepam 2,5 mg SOL VO & 6 & 24 \\
Flurazepam 30 mg CP VO & 1 & 4 \\
Lorazepam 1 mg CP VO & 2 & 8 \\
\hline
\end{tabular}

*CP - comprimido; VO - via oral; SOL - solução

0 fármaco clonazepam recebe destaque ao figurar em relatórios de Organismos Internacionais sobre as substâncias mais consumidas no mundo. 0 Conselho Internacional de Controle de Narcóticos (INCB), em seu relatório publicado no ano de 2009, apresenta indícios de um abuso sistemático de preparações farmacêuticas contendo clonazepam e aponta também a ocorrência do tráfico e do abuso da prescrição desse medicamento em muitos países. No Brasil, os dados relativos à comercialização do clonazepam apontam para um consumo superior a doze milhões de unidades deste medicamento entre os anos de 2008 e 2009 (MANGUINI, 2013).

De acordo com o levantamento disponibilizado pela ANVISAsobreoconsumo demedicamentospertencentes à Portaria no 344/1998, o clonazepam figura como o medicamento mais vendido no Brasil entre os anos de 2007 e 2010. Em 2007, foram dispensadas 29.463 unidades; em 2008, 4.784.730 unidades; em 2009, 7.498.569 unidades e em 2010, 10.590 .047 unidades, ou seja, houve um aumento de mais de $35.000 \%$ no consumo de clonazepam durante este período. Em um levantamento realizado no primeiro semestre de 2013, o clonazepam, ficou na 9a posição do ranking dos medicamentos mais vendidos no Brasil, sendo prescrito como ansiolítico em cerca de 93\% dos casos (ESERIAN; LOMBARDO, 2015).

Uma pesquisa publicada pela IMS Health, que é uma empresa especializada em pesquisa e fornecimento de conteúdo para a indústria farmacêutica, demonstrou que a escalada do benzodiazepínico Clonazepam no Brasil, que passou do sexto lugar no ano de 2001 entre os medicamentos mais vendidos passou para a segunda posição em 2008 (FOSCARINI, 2010), o que condiz com o presente estudo. Segundo critérios de Beers-Fick que classifica om medicamentos impróprios para idosos o Clonazepam é um medicamento de meia-vida longa que tem como possíveis consequências do uso sedação, possibilidade de quedas e fraturas (PASSARELI, 2006).

De acordo com a lista PRISCUS17 de medicamentos potencialmente inapropriados para idosos, adaptada à farmacopéia brasileira, que define os BZDs de longa ação como bromazepam, clonazepam e flurazepam são contraindicados para idosos pois são fármacos com risco de provocar efeitos colaterais superior aos benefícios em idosos (GORZONI; FABBRI; PIRES, 2012).

A utilização de medicamentos psicoativos em geriatria exige cuidados redobrados, tendo em vista que as taxas de metabolização de vários benzodiazepínicos declinam com a idade, tornando os idosos mais sensíveis 
aos efeitos adversos, particularmente os decorrentes do acúmulo do fármaco no organismo (DAL PIZOL; GRANZA; BASTOS, 2013). Há também uma diminuição da massa muscular, da água corporal, os mecanismos homeostáticos e a capacidade de filtração e de excreção também podem ficar comprometidos em idosos. Disso decorre a dificuldade de eliminação de metabólitos, o acúmulo de substâncias tóxicas no organismo e a produção de reações adversas (GALATO; SILVA; TIBURCIO, 2007).

Na Austrália, nos últimos 20 anos, a quantidade de BZDs em cada prescrição aumentou. 0 alprazolam se tornou a segunda droga mais popular, aumentando mais de oito vezes (BRETT, 2015).

Constatou-se no presente estudo que a maioria (52\%) dos entrevistados recebeu prescrição médica de um clínico geral representando 13 prescrições, seguido de 5 prescrições (20\%) de cardiologistas, 5 prescrições (20\%) apenas de psiquiatras e 2 prescrições (8\%) de ginecologistas.

Atualmente, 1 em cada 10 adultos recebem prescrições de BZDs a cada ano, a maioria por clínicos gerais (ASSOCIAÇÃO BRASILEIRA DE PSIQUIATRIA, 2006). A prescrição médica indevida também contribui para a manutenção do uso crônico de benzodiazepínicos. Grande parte dos consumidores recebe prescrições de clínicos gerais ou outras especialidades médicas, e não de psiquiatras ou neurologistas, que são os especialistas nestes tipos de prescrições, tendo em vista seu vasto conhecimento nesta área. Sendo assim, essa realidade propicia o surgimento de diversas complicações advindas do uso a longo prazo da medicação (PRADO FILHO, 2011).

A maioria dos problemas de origem psicológica ou psicossocial é vista primariamente pelo clínico geral, no atendimento primário. 0 médico da estratégia de saúde da família é o profissional atuante no nível de atenção primária a saúde no SUS e não é, necessariamente, um profissional especializado sendo na maioria dos casos um médico generalista. Pesquisa realizada na Espanha, em 1996, para investigar os padrões de prescrição de BZD dos clínicos gerais na área de captação de uma Unidade de Dependência de Drogas de um hospital mostrou que o médico generalista possui correto conhecimento sobre o uso de benzodiazepínicos, entretanto subestima capacidade do benzodiazepínico de causar dependência, assim como apresenta dificuldades para a descontinuação do tratamento (SCALERCIO, 2017).

Para Tancredi, o predomínio das prescrições provenientes de clínicos gerais, conduz ao uso indiscriminado destes medicamentos devido à falta de preparo para lidar com problemas psicológicos e existenciais, cedendo ao impulso de prescrever os tranquilizantes, muitas vezes subestimando a capacidade dos indivíduos em reagir às adversidades comuns aos processos vitais (TANCREDI, 1986). Se a prescrição de benzodiazepínicos é iniciada neste momento, e erroneamente, pode conduzir a um círculo vicioso que dura, muitas vezes, vários anos. Sendo assim, o conhecimento a respeito dos benzodiazepínicos e a sua prescrição correta por clínicos gerais são de suma importância (NORDON, 2009).

Em uma pesquisa realizada no Rio de Janeiro, sobre o consumo de psicofármacos, foi observado que o médico de clínica geral, ou especializado em áreas que não neurologia e psiquiatria, foi responsável, isoladamente, por $65,8 \%$ das prescrições. Os especialistas (neurologista e psiquiatra) foram responsáveis por $23,7 \%$ das indicações (ALMEIDA et al., 1994).

A orientação médica quanto ao uso dos benzodiazepínicos é muito importante para minimizar a incidência de efeitos colaterais. Os pacientes devem ser alertados sobre a ocorrência de déficit de atenção, riscos de dirigir veículos ou operar máquinas, risco de ingestão concomitante de álcool, bem como sobre o risco de desenvolvimento de dependência (CARVALHO et al., 2006).

Quando questionados os participantes da pesquisa quatro ao uso de bebida alcoólica, 9 pacientes (36\%) afirmaramfazer uso. Aorientação médica e farmacêutica sobre a interação com o álcool, dado seu intenso uso, também é muito importante, uma vez que pode ocorrer depressão respiratória grave e fatal pelo sinergismo do efeito depressor (AUCHEWSKI et al., 2004).

\section{Uso abusivo}

Quando questionados o que o médico informou quanto ao medicamento benzodiazepínico prescrito $64 \%$ dos pacientes só receberam a orientação que seria para ficar mais calmo. (Tabela 3) 
Tabela 3. Orientações médicas quanto ao medicamento a ser utilizado pelo paciente.

\begin{tabular}{llc}
\hline Qual informação que seu médico lhe passou? & N (25) & \% \\
\hline Para utilizar de forma contínua & 6 & 24 \\
Informações sobre riscos e benefícios & 1 & 4 \\
Utilizar eventualmente, a longo prazo pode causar diminuição da memória & 1 & 4 \\
Utilizar eventualmente pois pode causar dependência & 1 & 4 \\
\hline
\end{tabular}

Esta pesquisa também indica a carência de informação por parte dos usuários a respeito dos efeitos adversos ocasionados pelos benzodiazepínicos. Estudos realizados em outros países sugerem a relevância desta questão. Em uma pesquisa conduzida na Áustria, em que foram entrevistados pacientes internados que faziam uso de benzodiazepínicos, apenas $2 \%$ consideraram suficientes as informações providas pelo prescritor, enquanto $66 \%$ negaram ter recebido qualquer informação (FORSAN, 2010).

Em nove (36\%) dos pacientes entrevistados, o prescritor tentou reduzir a dose para retirar o medicamento, mas não obteve sucesso, pois os pacientes relataram sentir cansaço, insônia, agitação, ansiedade e tiveram que voltar com o tratamento e em dezesseis (64\%) dos casos o prescritor não tentou diminuir a dose ou retirar o tratamento, estes pacientes relatam que recebem prescrição para tomar como uso contínuo, a cada consulta, porém 5 (20,83\%) pacientes já tentaram interromper por conta própria o medicamento devido ao medo de causar dependência, mas não conseguiram e acabaram voltando ao tratamento pois sentiram cansaço, ansiedade e insônia, e esse poderia ter sido o motivo do insucesso.

Sabe-se que esses medicamentos devem ser interrompidos de forma gradual para evitar os sintomas de retirada que podem contribuir para que o paciente retorne a usá-los. 0 processo de retirada pode durar até seis meses. A prescrição deve ser avaliada sistematicamente pelo médico que assiste o paciente observando a indicação precisa, tempo de uso e as implicações decorrentes de seu uso prolongado, sempre com a aceitação do paciente. Geralmente, a retirada da primeira metade da droga é mais fácil que a retirada da porção final. É possível retirar os 50\% iniciais em um intervalo de 15 a 30 dias, e dos 50\% finais de modo mais paulatino (ASSOCIAÇÃO BRASILEIRA DE PSIQUIATRIA E NEUROLOGIA, 2013).
Apesar da segurança oferecida pelos BDZs, érelatada na literatura a recomendação preferencial de outras intervenções que não a prescrição de BDZ para o tratamento ou alívio sintomático de estados ansiosos e de insônia. São recomendados agentes farmacológicos não pertencentes à classe dos BDZ, bem como intervenções psicoterápicas ou combinação de ambos (ORLANDI; NOTO, 2005). Também pode ser aliada a abordagem farmacológica, intervenções vindas de grupos de autoajuda/apoio, sessões psicoterápicas e informações a respeito dos efeitos da substância utilizada e possíveis ocorrências durante o tratamento, sempre atendendo as necessidades de cada paciente (DIEHL; CORDEIRO; LARANJEIRA, 2016).

Experiências da terapia comunitária com mulheres tem sido uma estratégia adotada pelas equipes de saúde da família para enfrentar sofrimentos emocionais originados no cotidiano, proporcionando um espaço de falae departilha de situações inquietantes que provocam sofrimento emocional, para que as mulheres possam se sentir apoiadas pela equipe de saúde da família, e busquem estratégias mais eficazes de resolverem seus problemas sem recorrer primeiramente ao uso do psicofármacos. (FERREIRA FILHA, et al., 2009).

Entre os entrevistados, um paciente de 79 anos relatou fazer uso de mais doze medicamentos, outro de 77 anos faz uso de mais oito medicamentos, um paciente de 77 anos relata o uso de mais dez medicamentos e outro de 66 anos faz uso de mais 9 medicamentos, além dos benzodiazepínicos.

Segundo Rosenfeld o uso simultâneo de diversos medicamentos pode levar o usuário a fazer o tratamento de maneira incorreta se não houver acompanhamento e orientação adequada (ROZENFELD, 2003). Neste contexto, Romano-Líber e colaboradores (2002) descreveram a importância da intervenção do farmacêuticonouso de medicamentosBDZs, ressaltando que a informação fornecida ao usuário é tão ou mais 
importante que o medicamento por ele recebido, alcançando resultados promissores (LIEBER, 2002).

0 farmacêutico deve buscar atuar de forma mais efetiva na área de saúde mental. Em trabalho realizado no ano de 2009, sobre satisfação dos serviços farmacêuticos, o farmacêutico foi classificado pelos pacientes como a segunda melhor fonte para obter informações sobre medicamentos psicotrópicos e a população demonstrou ter uma percepção positiva dos farmacêuticos e dos serviços comunitários que estes podem fornecer (MARQUES; TIENGO; NOGUEIRA, 2013). É importante salientar que, mesmo em doses terapêuticas, esses psicofármacos podem levar à dependência. Isto é muito preocupante e comprova a necessidade de se assegurar o acesso aos medicamentos com segurança, eficácia e resolutividade, por meio da atividade farmacêutica comprometida com os princípios da promoção e prevenção de agravos da saúde (DE LIRA et al., 2014).

0 farmacêutico assegura que o paciente tenha acesso à informação sobre a utilização adequada dos medicamentos, o que contribui para o seu uso racional. Este profissional pode fazer o monitoramento da utilização dos medicamentos por meio da ficha de controle farmacoterapêutico, aconselhar acerca do uso de medicamentos de venda livre, sobre administração correta. A participação em programas de educação para a saúde em colaboração com outros membros da equipe de saúde e a construção de indicadores que visem mensurar a efetividade das intervenções buscando uma melhora na qualidade de vida e redução do uso desta classe de fármacos (ANDRADE; SILVA; FREITAS, 2004).

\section{CONCLUSÃO}

Por meio deste estudo pode-se definir que o perfil desses pacientes é composto de mulheres acima de 60 anos,com nível de escolaridadefundamentalincompleto, tempo de uso de benzodiazepínicos de 1 a 3 anos. 0 medicamento mais consumido entre as participantes foi o clonazepam, a maioria dos entrevistados não recebeu nenhuma informação sobre o medicamento como reações adversas, interação com álcool e dependência. A continuidade do uso vai além de uma finalidade específica e com um tempo indeterminado, demonstrando que há necessidade de conscientização dos profissionais envolvidos para o uso racional destes medicamentos.

A atuação do farmacêutico, no acompanhamento do uso correto do medicamento, é um fator crucial para o tratamento eficaz e seguro, seja com relação ao paciente ou com o profissional prescritor, informando ao paciente por quanto tempo deverá ser realizado o tratamento, doses recomendadas, possíveis efeitos colaterais que podem ocasionar tais fármacos, desempenhando papel de facilitador para o paciente e sua família, minimizando seu uso abusivo. Programas de atenção farmacêutica direcionados ao uso de psicotrópicos são essenciais para fornecer informação para os pacientes sobre os riscos da utilização desses medicamentos. 


\section{REFERÊNCIAS}

ALMEIDA, Liz Maria, et al. Consumo de Psicofármacos em uma Região Administrativa do Rio de Janeiro: A Ilha do Governador. Cadernos de Saúde Pública, Rio de Janeiro, v.10, n.1 p. 0516,1994 .

ANDRADE, Marcieni Ataide; SILVA, Marcos, Valério; FREITAS, Osvaldo. Assistência farmacêutica como estratégia para o uso racional de medicamentos em idosos. Semina: Ciências biológicas e da saúde, Londrina, v. 25 n.1 p.55-64, 2004.

ASSOCIAÇÃO BRASILEIRA DE PSIQUIATRIA E NEUROLOGIA. Abuso e Dependência de benzodiazepínicos. Associação Médica Brasileira, 2013. Disponível em: <https://diretrizes. amb.org.br/_DIRETRIZES/abuso_e_dependencia_de_ benzodiazepinicos/files/assets/common/downloads/ publication.pdf $>$. Acesso em: 05 nov. 2017.

ASSOCIAÇÃO BRASILEIRA DE PSIQUIATRIA. Diretriz: Uso Racional de Psicofármacos. Prefeitura do Rio de Janeiro: Programa de Saúde Mental, abr./jun. 2006.

AUCHEWSKI, Luciana, et al. Avaliação da orientação médica sobre os efeitos colaterais de benzodiazepínicos. Rev. Brasileira de Psiquiatria, v. 26, n. 1, p.24-31, 2004.

AUTHIER, N., et al. Benzodiazepine dependence: focus on withdrawal syndrome. Ann Pharm Fr., v.67, n.6, p.408-13, 2009.

BARReto, Lilia Cristina. Cuidados de Enfermagem ao paciente em uso de Benzodiazepínicos. 2014. 44f. Monografia (Especialização em Linhas de Cuidado em Enfermagem) - Universidade Federal de Santa Catarina, Florianópolis.

BRAGA, João Euclides Fernandes, et al. Ansiedade patológica: bases neurais e avanços na abordagem psicofarmacológica. Revista Brasileira de Ciências da Saúde, v. 14, n. 2, p. 93-100, 2011.

BRETT, Murion. Management of benzodiazepine misuse and dependence. Aust Prescr., v. 38, n.5, p.152-5, 2015.

CARVAlho, Andréia da Luz, et al. Uso Racional de Psicofármacos. 2006. Disponível em: <http://www.ensp. fiocruz.br/portal-ensp/judicializacao/pdfs/289.pdf>. Acesso em: 22 out. 2017.

CARVALHO, Lúcia de Fátima; DIMENSTEIN, Magda. 0 modelo de atenção à saúde e o uso de ansiolíticos entre mulheres. Estudos de Psicologia, Rio Grande do Norte, v.9, n.1, p.121-29, 2004.

CASTRO Gustavo Gomes, et al. Uso de Benzodiazepínicos como automedicação: consequências do uso abusivo, dependência, farmacovigilância e farmacoepidemiologia. R. Interd., v. 6, n.1, p.112-23, jan./mar., 2013.
DAL PIZOL, Tatiane da Silva; GRANZA, Maristela; BASTOS, Diego Coelho. Uso de Benzodiazepínicos em Hipertensos e Diabéticos de Passo Fundo, RS. Revista Contexto \& Saúde, v. 4, n. 06, p. 27-42, 2013.

CASTRO, L. A. P. G.; LARANJEIRA, R. Dependência de benzodiazepínicos. 2000. Disponível em http://www.uniad. org.br. Acesso em 01/12/2017.

DE LIRA, Aline Cavalcante, et al. Perfil de usuários de Benzodiazepínicos no contexto da atenção primária a Saúde. Revista de APS, Recife, v. 17, n. 2, 2014.

DIEHL, Alessandra; CORDEIRO, Daniel Cruz; LARANJEIRA, Ronaldo. Tratamentos Farmacológicos para dependência química da evidência científica à prática Clínica. São Paulo: Artmed, 2016.

ESERIAN, Jaqueline Kalleian; LOMBARDO, Márcia. Monitoramento da qualidade de comprimidos de Clonazepam distribuídos na rede pública Estadual de São Paulo e sua contribuição para o sucesso terapêutico. Eletronic Journal of Pharmacy, São Paulo, v. XII, n. 4, p. 57-64, 2015.

FERREIRA FILHA, Maria de Oliveira, et al. A terapia comunitária como estratégia de promoção à saúde mental: o caminho para o empoderamento. Revista Eletrônica de Enfermagem, Paraíba, v.11, n. 4, p. 964-70, 2009.

FORSAN, Maria Aparecida. o uso indiscriminado de benzodiazepínicos: uma análise crítica das práticas de prescrição, dispensação e uso prolongado. 2010.26f. Monografia (Especialização em Atenção Básica em Saúde das Famílias) Universidade Federal de Minas Gerais, Campos Gerais, MG. Disponível em: <https://www.nescon.medicina.ufmg.br/ biblioteca/imagem/0649.pdf>. Acesso em 22 out. 2017.

FOSCARINI, Priscila Tonial. Benzodiazepínicos: uma revisão sobre o uso, abuso e dependência. Universidade Federal do Rio Grande do Sul. Disponível em: http://www.lume.ufrgs.br/ bitstream/handle/10183/26847/000758691.pdf.Acesso em 02/10/2017.

GALATO Dayani; SILVA, Eduardo Souza; TIBURCIO, Leticia de Souza. Estudo de Utilização de Medicamentos em Idosos Residentes em uma Cidade do Sul de Santa Catarina: Um Olhar Sobre a Polimedicação. Revista Ciência e Saúde Coletiva, v.15, n. 6, p. 2899-2905, 2010.

GORZONI, Milton Luiz; FABBRI, Renato Moraes Alves; PIRES, Sueli Luciano. Medicamentos potencialmente inapropriados para idosos. Revista da Associação Médica Brasileira, Rio de Janeiro, v.58, n.4, p. 442-46, 2012.

HUF, Gisele; LOPES, Cláudia de Souza; ROZENFELD, Suely. 0 uso prolongado de benzodiazepínicos em mulheres de um centro de convivência para idosos. Cad. Saúde Pública, São Paulo, v.16, n.2, p. 351-62, 2000. 
IBGE. Instituto Brasileiro de Geografia e Estatística, 2017. Disponível em: <https://cidades.ibge.gov.br/xtras/perfil. php?codmun=430520>. Acesso em: 08 out. 2017.

LIEBER, Nicolina Silvana Romano, et al. Revisão dos estudos de intervenção do farmacêutico no uso de medicamentos por pacientes idosos. Cad. Saúde Pública, Rio de Janeiro, v.18, n.6, p.1499-507, 2002.

MAGALHÃES, Antônio Edson, et al. Psicotrópicos: perfil de prescrições de Benzodiazepínicos, Antidepressivos e Anorexígenos a partir de uma revisão sistemática. Eletronic Journal of Pharmacy, v. 13, n.3, p. 111-122, 2016

MANGUINI, Zacarias Antônio. Condicionantes relacionados ao uso crônico de Clonazepam no Brasil: uma história de vida. 2013. 91f. Dissertação (Mestrado) - Universidade Federal de Santa Catarina, Centro de Ciências da Saúde. Programa de Pós- Graduação em Saúde Coletiva, Florianópolis, SC.

MARQUES Luciene Alves Moreira; TIENGO, Alessandra; NOGUEIRA, Valéria Aparecida. Avaliação do uso de benzodiazepínicos por clientes de uma drogaria privada. Rev Univ Vale Rio Verde, Três Corações, v.10, n. 1, p. 234-44, 2013.

MARQUES, Fabricio Correa. Estudo transversal relacionado ao uso de benzodiazepínicos no Centro de Atenção Psicossocial (CAPS) do município de Campo Bom - RS. Universidade Federal do Rio Grande do Sul. 2015. Disponível em: <http://www.lume.ufrgs.br/handle/10183/140022 2. Acesso em: 15 out. 2017

MENDONÇA, Reginaldo Teixeira, CARVALHO, Antônio Carlos Duarte. 0 consumo de benzodiazepínicos por mulheres idosas. SMAD, Rev. Eletrônica Saúde Mental Álcool Drog. (Ed. port.) v.1 n.2 Ribeirão Preto ago. 2005.

NOIA, Aparecida Santos, et al. Fatores Associados ao uso de psicotrópicos por idosos no Município de São Paulo. Revista da Escola de Enfermagem USP, São Paulo, v. 46, esp, p.38-43, 2012.

NORDON, David Gonçalves, et al. Características do uso de benzodiazepínicos por mulheres que buscavam tratamento na atenção primária. Revista de Psiquiatria do RS. v.31, n.3, p.151-158, 2009.

NORDON, David Gonçalves; HUBNER, Carlos, Krakauer. Prescrição de benzodiazepínicos por clínicos gerais. Diagn Tratamento, Sorocaba, v.14, n. 2, p.66-9, 2009.

ORLANDI, Paula; NOTO, Ana Regina. Uso indevido de benzodiazepínicos: um estudo com informantes-chave no município de São Paulo. Revista Latino Americana de Enfermagem, São Paulo, v. 13, p. 896-902, set./out. 2005.
PAPROCKI, Jorge. 0 emprego de ansiolíticos benzodiazepínicos pelo clínico geral e por especialistas não psiquiatras. Arquivos Brasileiros de Medicina, Rio de Janeiro, v. 64, n. 5, p.305 1990.

PASSARELI, Maria Cristina. Medicamentos inapropriados para idosos: um grave problema de saúde pública. Boletim Informativo Farmacovigilância, São Paulo, 2006. Disponíve em: < http://www.cvs.saude.sp.gov.br/zip/bfarmaco_2.pdf> Acesso em: 15 out. 2017

PRADOFILHO,PauloCelso, etal.Utilizaçãodebenzodiazepínicos por idosos de uma estratégia de saúde da família: implicações para enfermagem. Esc. Anna Nery, Rio de Janeiro, v.15, n.3, 2011.

RODRIGUES, Maria Aparecida; FACCHINI, Luis Augusto; LIMA, Maurício Silva. Modificações nos padrões de consumo de psicofármacos em localidade do Sul do Brasil. Rev. Saúde Públic, São Paulo, v. 40, n. 1, jan./fev., 2006.

ROZENFELDT, Suely. Prevalência, fatores associados e mal uso de medicamentos entre idosos: uma revisão. Cad. Saúde Pública, Rio de Janeiro, v. 19, n.3, p.717-24, 2003.

RUFF, Gisele. et. al. O uso prolongado de benzodiazepínicos em mulheres de um centro de convivência para idosos. Caderno de Saúde Pública, Rio de Janeiro, v.16, n. 2, p.351-62, 2000.

SCALERCIO, Priscila Lima de Araújo. Avaliação do uso de benzodiazepínicos em um município de médio porte do estado do Paraná. Universidade Federal do Paraná, Curitiba 2017.

SILVA, Eliane Regina Biasi; OLIVERIA, Karla Renata. Estudo da utilização e promoção do uso racional de benzodiazepínicos em uma drogaria no município de São Luiz Gonzaga - RS. Rev. Bras. Farm., v.93, n. 2, p.153-60, 2012.

SOUZA, Ana Rosa Lins, et al. Contextos e padrões do uso indevido de benzodiazepínicos entre mulheres. Revista Ciência e Saúde Coletiva, Rio de Janeiro, v.18, n. 4, p.1131-140, 2013.

TANCREDI, Francisco Bernardini.Consumo de medicamentos benzodiazepínicos no Brasil - 1970 a 1985: análise comparativa de tendências. 1986. 273f. Tese (Doutorado em Saúde Pública) - Universidade de São Paulo, São Paulo.

WANDERLEY, Tyago Costa; SANTOS, Silvana Cristina. Uso de Benzodiazepínicos e suas implicações: revisão integrativa. Rev Enferm UFPE online, Recife, v. 9, n. 8, p. 8865-73, 2015. 